Hospital de Niñas Manuel Arriarán.

Catedra Extraordinaria de Clinica Pediátrica

Prof. Eugenio Cienfuegos.

\title{
COMOLIACIONES CeREBRALES AGUdAS DE LAS GLOMERULONEFRITIS
}

PCi la: Dies. JUL 10 MENEGHELlo R. (*) y HERMANN NIEMEYeR $F$.

El estudio de las glomérulo-nefritis infantiles viene siendo motivo de especial preocupación para la cátedra del Prof. Cienfuegos y tiesde hace 15 años el probiema ha sido abotdadado desde diversos aspectos que han quedado establecidos en numerosas publicaciones. No es sólo la frecuencia de esas afecciones lo que ha generado aquella preocupación, sino su modo de evolucionar, sus caracteres etiopatogénicos singulares que han dado origen a hipótesis originales respecto a su génesis. sino que támbién, junto con valorar la experiencia recogida en otros centros de estudio, se procura cada día revisat las anteriores invest:gaciones con el fin de llegar a mayores progresos en cuanto se refiere a dietética y tratamiento.

En los ya numerosos trabajos sobre este tema, Cienfuegos y colaboradores $(1,2,3,4,5,6)$ han investigado tópicos variados respecto a la más frecuente neofropatía inIfantil: en la exposición de los casos que motivan el presente trabajo nos vamos a limitar al análisis del episodio cerebral que han solido presentar los enfermos en el curso de su glomérulo-nefritis. Omitiremos los deta'les quel se refieren a 13 "volución de la enfermedad misma, ya que será mayormente analizada pronto en otra comunicación qué abarque el estudio de los enfermos atendidos durante el año 1943. Todos estos niños han sido sometidos a un plan de investigación que comprende diversos exámenes:

(*) Ayudante de la Cátedra de Clínica Pediátrica del Prot. Cisnfuegos. 
1. Medición de la presión arterial tres veces á día durante el periodo hipertensivo $y$ después una vez en las mañanas.

2. Medición de la diuresis y densidad de ia orina diariamente.

3. Constante de Ambard e indicá de depuración uréca, al ingreso y después de cada diez dias.

4. Examen ce orina con recuento de gióbulos rojos. cada cinco días.

5. Examen de fondo de ojo, al ingreso y después cada diez días.

6. Peso diario.

7. Examen otorrinolaringo'ógi:o y dental.

8. Proteinemia al ingreso y cada diez dias.

9. Examen bacteriológico de !a secreción faríngeta cada diez dias.

10. Examén parasitológico de las deposiciones; cada diez días.

1:1. Cuando el enfermo está próximo a ser dado de alta: se agrega la prueba de Vo'hard-Fishberg y'ell reuento de Addis.

12. La alimentación, de acuerdo con la experiencia ya analizada en varios años y expuesta en trabajos anteriores. ha sido el régimen normal del hospital, sin restrictión alguna relspecto a líquidos, sales y proteinas, regida sólo por el apetito del enfermo. Como tratamiento medicamentoso. hemos usado en estos casos el sulfato de magnesio por via intramusculat o bucal siguiendo las técnicas dictatas por experiencias anteriores del Servicio.

En este trabajo se hace el análisis de a!gunos casos presentados eln 1943 en el Hosp:ta: Manuel Arriarán de lo que Blackfan (7) llama "complicaciones cerebrales agudas" de la glomérulo-nefritis y que corresponden a lo que se ha denominado uremia eclamptica. pseudouremia aguda o uremia convuísiva. Nos parece acertada la nomenclatura propuesta por Blackfan, pues no alude a la patogenia del accidente y evita lá confusión con la genuina uremia originada por insuficiencia renal.

La frecuencia con que se presentan las complicaciones cerebrales agudas en el curso del la gloméralo-nefritis aguda es muy variable, según los diferentes autores. En "Archives de Médicine des enfants", de 1937, aparece una estadística en la que se hace notar que de 310 niños recibidos en el HôpitaÍ des Enfants Malades en el curso del 5 años y medio. 
por nefritis aguda, 23 tuvieron convulsiones, o sea un $7,4 \%$. Aldrich (8), en 1931, cita 28 casos. Galasso (3) encontró un solo caso con convulsiones entre 49 nefritis. Baetza Goñi (5) muestra 4 casos en su estadíst ca de 893 observaciones revisadas. Durante el año 1943 se hospitalizaron eln el Arriarán 66 nefritis agudas, de las cuales 5 enfermos presentaron complicación cerebral.

'El cuadro clínico es variabla de un enfermo a otro, pero tiene rasgos comunes, que es conveniente destacar. En general, las complicaciones cerebrales agudas aparecen en los primeros dias del comienzo aparente de la glomérulo-nefritis o en pleno curso de ésta cuando ha sido "mal tratada". Varela (9). Son frecuentes en la nefropatía gravidica, raras en la nefroesclerosis retráctíl y muy excasas en anurias no nefríticas Ascoli. Muller y Volhard las han observado en anurias por cclusión de los uréteres. Gantes (10), entre otros, cita un casc de eclampsia en un niño con dispepsia y edema generalizado que presentó anuria. Importante es hacer notar que los nefrósicos muy rara vez presentan complicaoión cerebral.

El sintoma más espectacular de la complicación es el ataque convalsivo. Este puede presentarse súbitamente, pero lo corriente es que vaya precedido de signos prodrómicos, entre los cuales ocupa un lugar preferente la cefalalgia, que en ocasiones se irradia a la nuca produciendo rigidez de ésta: además existén náuseas, vómitos, apatía progtesiva, vértigos. visión borrosa: puede haber somnolenc'a o insomnio con gran intranquilidad. La piel está seca y los reflejos tendinosos exaltados. A pesar de que pueden faltar, la regla es que se encuentren los sintomas de la glomérulo nefritis, es decir. presión arterial elevada, edemas y sindroma urinario. El más constante es la hipertensión. Importa hacer notar que cuando aparecen los profrontos durante la evolución de una glométulo nefritis aguda, es frecuente observar una nueva elevación de la presión artitrial dentro de! a!za estable $v$ definida de ella y que la hace !'egar a cifras muy altas. Este hecho aonstituye una razón no despreciable oara que ell médico tome la presión varias veces al día en los nefriticos agudos hipertensos. ya que podría eljercer una acción profiláctica de la complicaciọn cerebra!.

A continuación de estos prodromos, que suelen durar algunos días, y aue pueden pasar inadvertidos o set muy llamativos, estalla el acceso convulsivo, buscamente o después de haber caido gradualmente an coma. El gran ataque tiene gran similitud con el ataque epiléptico. No es raro que sea 
precedido de un grito. Luego se produce la pérdida del conocimiento, que es seguida de violenta contracción tónica de toda la musculatura, determinante det ligero grado de opistótono y apnea. La cara pálida y edematosa típica del renal, toma colot rojo-azulada, con ingurgitación venosa del cuello. adquiriendo las fascies aspecto de terror. A los pocos segun. dos se inician las contracciones clónicas desordenadas, en que participan también todos los grupos musculares inclusive los de la cara. La respiración es muy irregular. Las convulsio. nes cesan poco a poco hasta llegar a la relajación completa. El enfermo queda inconsciente, con respiración etstertorosa, por un tiempo variable, para luego pasar a un sueño profundo, del cual al poco rato se le puedet despertar. Al principio reacciona perezosamente y se muestra indiferente a lo que lọ rodea, pero más tarde su sensorio se despeja, quejándose con frecuencia de cefalea.

No siempre, por cierto, el ataque eclámptico se ciñe a este esquema. Así, aunque el coma es la regla. hay casos en que el enfermo conserva la conciencia durante sus convulsiones (Bright) (14 a). Las contracciones pueden iniciarse por contracciones fulgurantes en el territorio del facial, que se extienden luego a todas las extremidades o bien a un solo lado, tomando entonces el aspecto de típica epilepsia jacksoniana. Chauffard (14 b) observó un caso en que las convulsiones se alternaban rápidamente de un lado a otro del cuerpo. 'También se' han descrito caros raros con contracciones de tipo coreico, atetósico o tetánico. En esta últíma circunstancia con Chvosteck y Trousseau positivos (jposibie coexistencia de nefritis con hipocalcemia?). A veces puede presentarse un verdadero "estado eclámptico" en que - persistiendo o no el estado de coma - se suceden varios estadios convulsivos. Se han descrito hasta 100 y 200 accesos en 24 horas.

Durante el ataque los reflejos tendinosos están exaltados y muchas veces all Babinsky es espontáneo. Hay midriasis con reacciones pupilares flojas o abolidas; el examen de fondn de ojo demuestra un ectasis papilar. La tempertatura corporal generalmente sube, aunque a veces es subnormal. Volhard da gran importancia a la midriasis y a la hipertarmia, en $\mathrm{e}^{1}$ diagnóstico diferencial con la uremia verdadera, en aue por lo general existen miosis $\mathrm{v}$ tendencia a la hipotermia. El vulso durante el ataque está tenso y acelerado. siendo ous en el periodo prodrómico estaba más bien lento. Aunquz no 
es lo normal, durante el ataque puede perdersel el control esfinteriano, echar espuma por la boca y aun morderse la lengua. La rápida subida del la tensión arteriá, junto a la disminución de la circulación coronaria por contracción arteriolar, y al mayor esfuerzo circulatorio ceterminado por las convulsiones, pueden causar nsuficiencia aguda del miocardio, con caída secundaría de la presión arterial y muerte rápida por falla circulatoria.

La presión del líquido céfalo raquídeo por lo general elstá elevada, aunque a veces es norma:. En la sangre, comúnmente no se observan grandes alteraciones, salvo el caso en que se agregue insuficiencia renal, que se acompaña de uremia alta.

La orina muestra el sindrome típico de la glomérulo nefritis aguda. A veces precede al ataque una acentuada disminución de la diuresis.

Pasado el ataque suele presentarse con cierta frecuencia la amaurosis y que a vecies constituye ella sola la complicación cerebral de la giomérulo neffritis aguda. Los trastornos auditivos son raros. (Rosenstein) (14 c).

Después del ataque pueden quedar trastornos psíquicos. estados crepusculares afinés a los epilépticos, amnesia retrógrada (por ejemplo. en la eclampsia del parto).

Al lado de formas cerebrales con predomin:o motriz se describen formas en que predomina el cuadro de las perturbaciones psíquicas. En general son raras. Se presentan delirios, alucinaciones, psicosis de corta duración, pues se recuperan pronto o van a la muertel. Los casos descritos antiguamente de larga evolución son sin duda de otro origen.

La patogenia de las complicaciones cerebrales agudas de la glomérulo nefritis aguda dista mucho de estar aclarada. Las hipótesis tendientec a expl carla se basan más en esquemas especulativos gue en ta realidad clínica o experimental.

1. Durante un tiempo se atribuyeron los síntomas cerebrales a la insuficiencia renal. aue producía una retención salina (Widal (11) y Ambard (12) o ureica, capaz de intoxicar la célula cerebral. Pero Volhard (14 d) (1918). y estudios posteriores demostraton que hay integridad de la función renal y fálta de retención de los constituyentes urinarics, aunque for supuesto, pueda haber coincidencia de una insuficiencia renal con el estado convulsivo.

2. Se ha dado mucha importancia al edema cerebral. que con frecuencia acompaña esta complicación. Este edem podría actuar de dos maneras: comprimiendo los pejueños 
vasc: 5 (Traube) (14 e) y determinando así anemia encefálica, obien, produciendo una compresión cerebral contra la caja craneana no distensible (Volhard) (14 d). Pero estei modo de pensar ha sido rechazado debido principalmente a los argumentos de Lichtwitz y Cohnheim (14 f).

3. Hoy se tiende a colocar en primer plano el factor constricción de las arteriolas cerebrales. Fishberg (14), al revisar el problema aporta diversos argumentos de orden clinico y experimental en apoyo de la hipótesis que afirma que lo fundamental es la constricción vascular, la cuar determinaria isquemia cerebral. Esta sería en última instancia la rezponsable de alteraciones metabólicas que se irían a manifestar como e! cuadro del ta complicación aguda cerebral.

El diagnóstico se hace sin grandes dificultades si hay antecedentes de glomérulo nefritis. En enfermos que l'iegan sin antecedentes y durante eil ataque, se plantea la cuda diagnóstica con diversas afecciones: epilepsia, meningitis, tumor. absceso o.trombosis cerebral, hipelrtensión esencia:, etc. Los siguientes elementos ayudarán a resolverla:

l. Examen de orina: especialmante buscar hematuria. ya que en la epilepsia suele presentarse una albuminuria que persiste varias horas después del ataque.

2. Presión arterial, dato muy valioso por la constan: cia de su alza. En el tumor cerebral suele haber hipertensión. pero no tan marcada.

3. Punción lumbar, que junto con aliviar al paciente, descarta la posible meningitis y la hemorragia subaracnoidea. Es muy constante 'a hipertensión del L. C. R., fenómeno que ocurrel también en los tumores cetebrales, en las meningit:s $y$ en ciertos casos de hipertensión en fase maligna.

Pronóstico. Pasado el ataque, y con los tratamientos modernos, el pronóstico es muy bueno. Aldrich (13) establece que en su clínica no hubo ningún caso fatal entre 35 casos tratados. Fishberg (14) dice que la expertiencia en la guerra de 1914 mostró que la recuperación era completa en una gran proporción de los accesos convulsivos th las nefritis de la guerra. En la práctica civil se ha observado to mismo en nefritis, postescallatinosas y de otro t po. Sin embargo. la seguridad no es abso'uta mientras dura ell ataque, pues el paciente puede morir súbitamente por'falla circulatoria. o por parálisis bulbar, aun durante la primera convulsión. Si las convulsiones se repiten con cortos intervalos $e_{1}^{1}$ pronóstico. es más serio. 
Respecto al pronóstico final de la enfermedad, todos los autores están de acuerdo en que la aparición de la complicación cerebral no tien infuencia en el curso del proceso renal.

En el tratamiento se ha recomendado el uso de las siguientes medidas:

1. Regimen normal con cierta restricción en las proteínas (13). En realidad puede indicarse la alimentación que se desee ya que gneralmente el enfermo come poco y sólo pide agua. tación.

2." Reposo, abrigo y obscutidad, evitando toda exci-

3. Punción Iumbat, que a veces es de efecto espectacular (14).

4." Sangría de 200 a $300 \mathrm{cc}$ de acuerdo con la edad del paciente. Mecanismo no bien explicado.

5. Sulfato del magnesia por vía oral. intramuscular o endovenasa. En el primer caso se emp'ea solución al $50 \%$. en cantidad que varía entre 20 y 40 cc. cada 4 horas según el grado de hipertensión. Puede sólo instituirse si el enfermo está consciente. La vía intramuscular es la más usada entre nosotros aunquet tiene e! inconveniente de ser muy dolorosa. Se emplea al $25 \%$ en dos's de 1 cc. por año de edad, repetida con frecuencia variable (cada 3,4 ó 6 horas). La vía endovenosa, que nosottos no hemos practicado, se emplea usando la solución al $2 \%$ en cantidad de $10 \mathrm{cc}$. por kilo de peso. Por lo común se colocan 100 a $300 \mathrm{cc}$. de la solación al $2 \%$. El efecto del sulfato de magnesia bien empleado es sorprendente, pero hay casos en que fracasa. Sel puede recurrir entonces a los enemas con brumuto de calcio e hidrato de cloral, a la anestes a con cloroformo y si las convulsiones son intensas e incontro'adas por los métodos anteriores se recurre a la morfina.

6.2 Algunos autores (14) han preconizado e1 uso de soluciones bipertónicas del glucosa o sucrosa.

El mecanismo de acción de tóca la terapéutica ind cada ha sido interpretado de diferentes maneras, según la teoría etiopatogénica de la complicación cerebral aguda.

\section{Casos clínicos.}

OBS. 70724 , - G. R. 7 años.

Ingresa con un cuadro iniciado dos meses y medio antes, en la convale. cencia de tina varicela, con edema de las extremidades, que luego ee generaliza: cefalea orinas oseraras y escasas. Por indicación médica guarda cama algunos dias $y$ se levanta en mejores condiciones aparentes. Cinco dias antes del ingreso 
al hospital, presenta vómitos, cefaleas Y un ataque convulsivo con pérdida del conocimiento. Es tratado con sulfato de magnesio (se ignotan las dosis) y suero glucosado hipertónico, con lo que se atenúan los sintomas. Sin embargo, $1_{2}$ agudeza visal empieza a disminuir francamente $y$ las orinzs a hacen hematúricas. por lo cual se deciden a bospitalizarto. En receprión se cneuentra un niño consciente, pálido, febri] $\left(38^{\circ}\right)$. Pulro regular, tenso, 130 por minuto, Presiós, 130-110 (Bümanómetro). Anasarca, Disminnçión muy marcada de la risión, Ruido de galope. El examen de orina tevela 9.6 gramos por mil de albúmina y glóbulos rojos muy abundantes. Uremia, 0.32 por mil. Fondo de ojo normal. I.a ceguera fá aumentando hasta alcanzar amaurosis complata el tercer día, con psiquis embotado $y$ respiración de Claynes-Stokes. Tonos cardiacos bien timbrados. iaquicardia. Se mantiener el anasarca y la presión elevaja. Al quinto dia de ingrisso, después de un descenso del pezo de alrededpr de 2 kgrs.. récupera bien la visión y está consciente. El fonło de ojo, controlado diatiamente. revela siempre notmalidad. El tratamiento consistió en inyecciones intramusculares de sulfato de magnesio al $25 \%$ y per os al $50 \%$. Régimen normal durante dos días yuz despoés se cambió por régimen normal sin sal y liquido controlado.

Permaneció 50 dias en el hospical. en el transcurso de los cuales no volvió a presentarse episodio convulsivo. La evolución fué desfavorable, pues la presión se mintuvo elevada. los examenes de orina revelaron pirsistencia de albaminuria (alrededor de dos por mil) y hematuria, y las pruebis de fanción rensl siempre esruvieron altetadas. - A solicitud insistente de Ja madre faé dado de alta, pcro volvió a control a los 15 dias. En esta ocasión. el niño sencontraba en buen estado general aparente, pero el sirdrome urinario continuaba igual, 1 a presión seguía alta (120 a 130 la máxima) y las prosbas funcionales malas. La amigdalectomía no cambió estas circunstancias. De nuevo hubo que darlo de alta a pedide de la familia.

OBS. 62069. - M. M. 12 años.

lngresa el 27 de mayo de 1943. Sa enfermedad s: inicia mis o mathos al zí́a 23 con decaimiento. tos y arinas escuras. Tres dias despnés presenta escalofríos y cefalea intensa. Seis boras antes de su ingreso se queja de disminución de la visión y cuatto horas más carde presenta ataque convulsivo generaltzado, por lo cuai recurre a la Posta de Urgencia. En el examen físico dz ricepción se encuentran los siguientes hechos de importancia: palidez intensa de los tegamentos: edema: t3quicardia, psiquis embotado, estado febril, estartores bronquiales diseminados en ambos campos palmonares. convulisiones aisladas de los miembros, presión atterial 150-100. Se hospitaliza con el diagnóstico do complicación cerebral aguda en una glomérulo nefritis aguda; bronquitis. Se practica una sangria de 300 c.e. no obteniéndose con esta medida ninguna modificación del cuadio clínico. Además, se coloca solfato de magntsia, 10 c.c. al $25 \%$ intramuscular. Al cabo de cuatro horas, el enfermo estílúcido, y se que- 


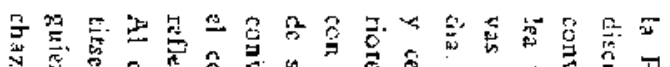

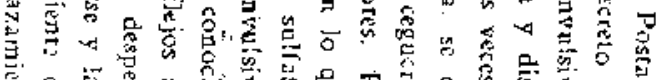

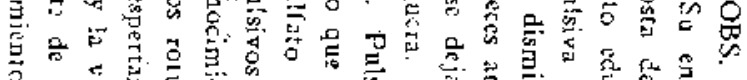

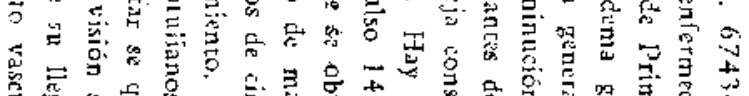

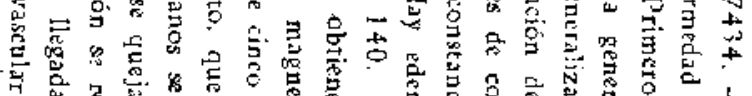

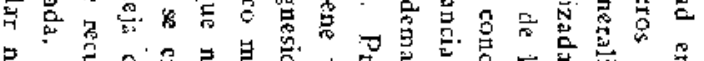

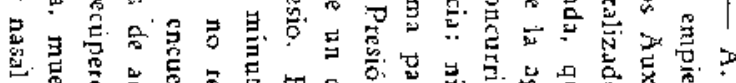

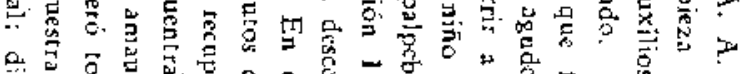

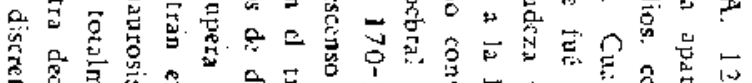

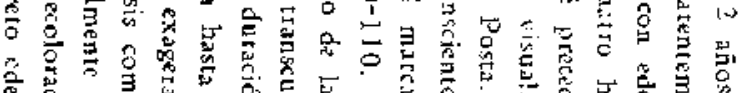

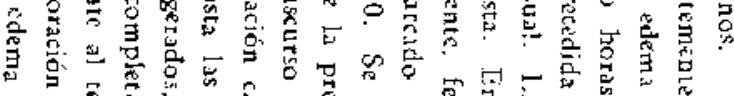

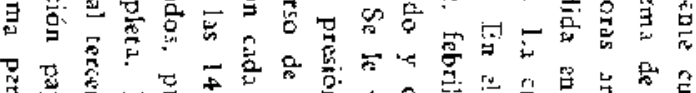

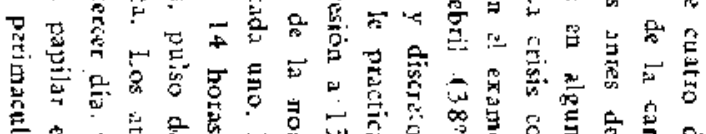

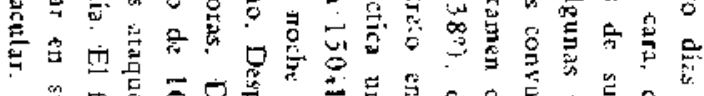

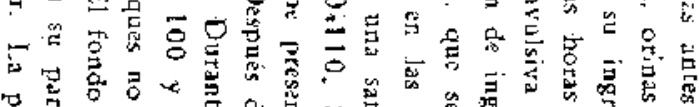

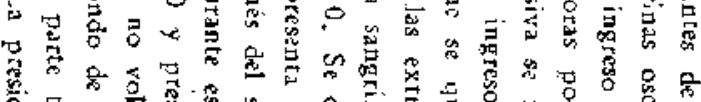

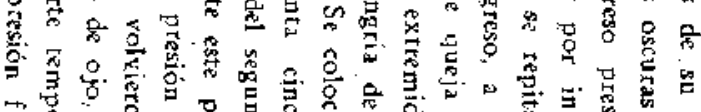

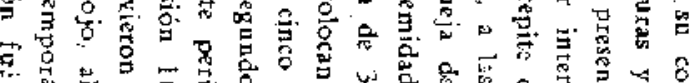

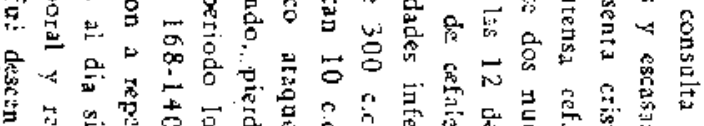

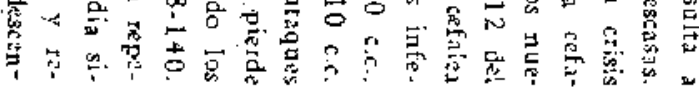

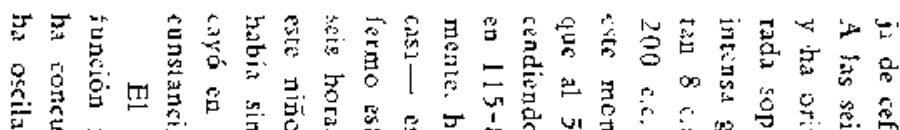

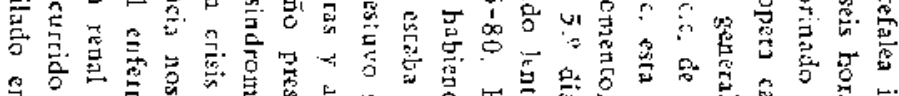

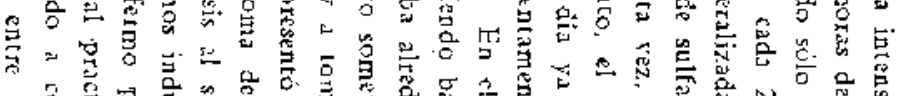

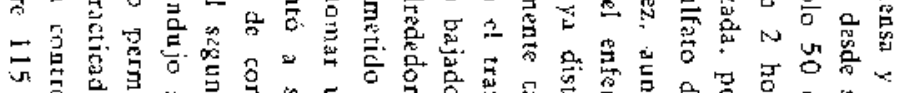

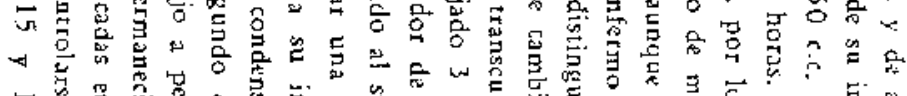

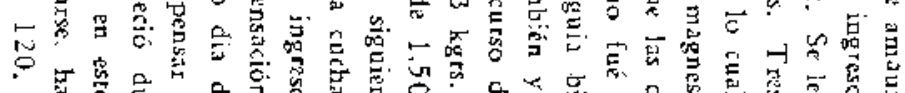

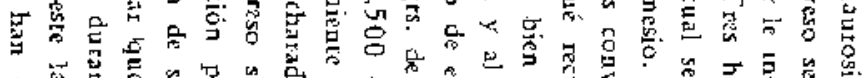

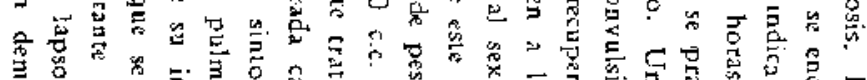

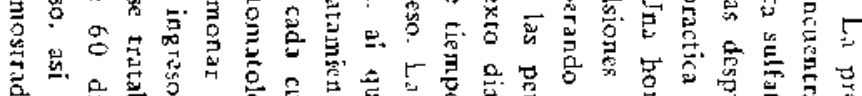

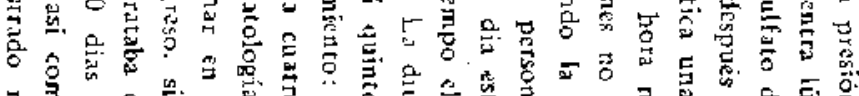

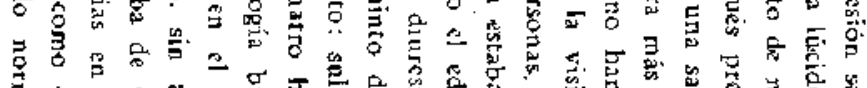

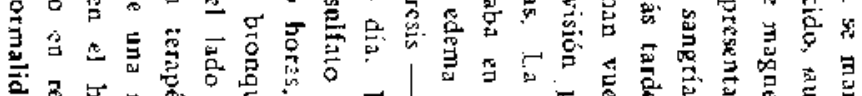

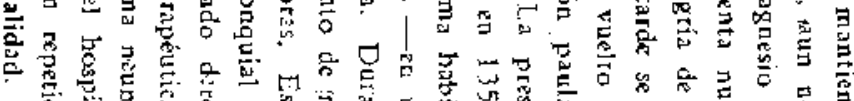

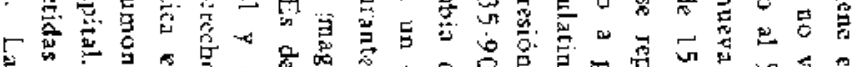

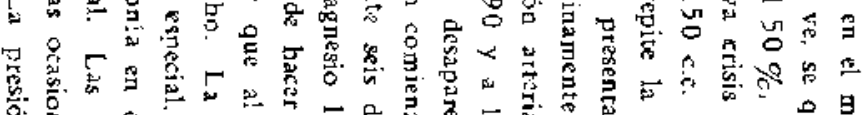

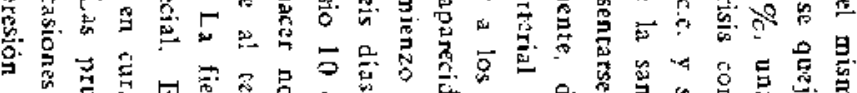

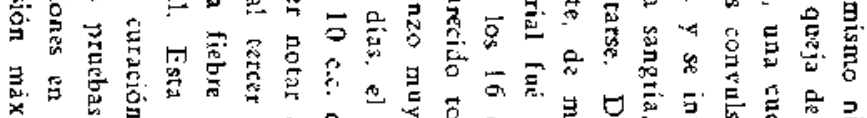

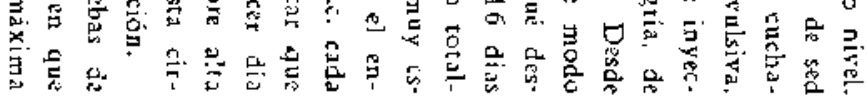


diendo lentamente, de modo que al quibto día era notmal. En diez dias el edema se había fundido ('baja de peso $2.5 \mathrm{kgrs.}$ ). La uremia, al ingreso, era de 0.45 por mil y en exámines posteriores osciló entrz 0.40 y 0.30 . Permaneció bospitalizado durante 47 dias. Las pruebas de función renal, practicadas al ser dado de alta $y$ rapetidas periódicamente como control. han cido normales.

OBS. 63830. - M. V. 11 años.

Una semana antes de su llegada al hospital lo notan con la cara edematos. inaperente. Cuatro horas antes del ingreso (a jas 15 horas). presenta ataque convulsivo generalizado, con fírdida det conocimiento y expulsión de espuna pist la bocs, que duró ¿uaa hora? Después del ataqu: queda inconsciente $y$ tres horas después sa repité con mordedura de la lengua. En el Sirvicio da urgencia te encuentra niño incensciente, febril $\left(: 8^{4}\right)$. con axcitación motora, estado con: tractural de ilas extremidades inferiores, midriasis. etema de la cara. Puiso 100. regular. Respiración, 80. Presión, 140-80. Se colcan enema de bromuto con cloral; sulfto de magnesio, 6 c.c. intranusular. Cotro el cuadro no cede, se practica uns anestesia general etćrea $y$ un momento despućs se hace una sangria de 200 c.c. por persistir la excitación motora. Queda en estado di somnolenzia

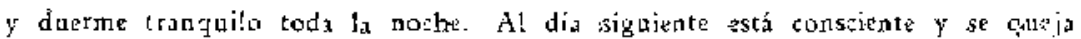
de cefalea $y$ de disminución de la agudeza visual, El examer neurológico acusa normalidad de los reflejos. El fando de ojo es negarivo. La presión sanguíned es de 165-110. I_s uremia de $0.30 \mathrm{gr}$. por mil. Al cereer dia la presión arerial descendió y se mantuvo alrededor de 110-70 durante los 43 dias de estatía del enfermo en el hospira?. El edema de la cara desapateció al tercer o cuarto día. El sindrome urinatio. muy atenuzdo en un principio. desaparció al quinto dia. El trataminto se hizo a base dix régimen normal $y$ sulfato $d e$ magnesio incramuscular (mientras duró la hipertensión). La evolución fué hacia $l_{a}$ mejoría completa. szgún lo demuestran los controlss hechos dospaés de sti alta.

CBS. 61746. - R. A. 12 años.

Alred:dor del 7 de marzo de 1943 aparece edena ficial, de la pared atcominal $y$ de las extremidades inferiors. Junto a esto; dacaimiento y anore$x$ ia. La sintomatologia descrita va en amento y luego si agregan oliguria, hematuria y vémitos. E1 11 de marzo en la nocbe. pierde ei conocimiento y pocas horas después, presenta convulsiones gencralizadas, quo duran hasta las seis de la madragata. Liega a esa boca a la Posta de primaros auxilios en estado inconsciente, con convalsiones de la cara y extremidad supesior direcha, mistagraus horizontal, pulso 78 , discteto edema de la pared abdominali presión arteral 160-115. Uremia $0.70 \mathrm{gr}$ por mil. Albuminuria 11 gis. por mil y hemacuria. La Juación Ímbar da salida a líquido gota a go:a cluro. aibúmina 0.60 , sloruros 7.35. glucosa 0.90 , Pundy +++ . Se diagnostica complicación cerebeal en uni glomérulo neftitis aguda y se hake una sangria de 250 c.c. Se indica sulfato de magnesio, 10 c.c. intramusiulat cada cuatro horas $y$ régimen de hambre con testrición de liquidos. Algunas bora; más carde a pesar di 
Ia terapética instituida, continúan las convalsiones localizadas al lado derecho del cuerpo, comprendiendo cara, brazo y piesna, de tal violencia, que bay nzcesidad de dejar una enfermera a permanencia a su lado, para quo no cayera de la cama. En este momento, el fondo de ojo es negativo, la temperatura es de $38^{4}$, las pupilas reaccionan bien a la luz y por momentos aparece nistagmus horizontal. Hay conțrol esfinteriano. Se coloca un enema de bromato ton clo13. Dos horas después cesan las convulsiones, la presión ha descendido a 135 90 y el enfermo se encuentra en estado sopotoso. El resio de ta tarde continúa indiferente al medio. sin responder a las preguntas que se le hacen. Dutante la nocbe duerme tranquilo $p$ al dia siguiente amanece lúcido, quejädose de sed intensa. La presión arterial es de 160-100. Se lo diaja con tégimen normal. Durante seis dias continúa con cierta torpeza mental. La presión se mantienc alta. l!egando en una ocasión a 190-130. Al cabo de este tiempo se produce aumento de 12 diuresis con fusión de edemas y la presión tiende a normalizarse. La uremia baja a ciftas nomales y el sindroma urinatio se atenúa. Patmanecte en e! Servicio 57 dias y la evolnción fué favorable, de acuerdo con los resultados de las pruebas de la función reral.

\section{Biblíografía.}

1.-CIENFUEGOS E. - Rev. Chil. de Ped. IV, 69; 1943.

2.-URETA E. - Rev, Chil. de Ped. IV. 98, 1933.

3.-GALASSO W. - Rev. Chil. de Ped. Xl, 115, 1940.

4.-OSSA R. - Tesis 1941.

5.-BAEZA GONI A, -- Glomérulo nefritis de la infancia. Ed, Zig-Zag. Santiago de Chile, 1942 .

6.-BURGOS S. $\rightarrow$ Tesis 1943.

7.-BI.ACKFAN K. D. and MAC KAHN C. F. - J. Am. M. Ass. 97: I052, 1931 .

8.-ALDRICH C. A. - Am. J. Dis, of Child. 41: 1265, 1931.

9.-VARELA M. - Nefropatías. Ed. Atenzo, Buenos Aires, 1943.

10.-GANTES R. - Atcb. Ho:p. R. del Rio, marzo 1930.

11.-WIDAL y JAVAL. - La cure de décloruration. Patís, ' 1906.

12.-AMBARD y SCHMID. - La réserve alcaline. Paris, 1928.

13.-ALDRICH C. A. - Brememann's Practice of Pediatrics.

14.-FISHBERG A. M. - Hypertension and nepbritis. Lea and Febiger. P.r. 1940 .

a) Btight, citado por Fishberg.

b) Chauffard, citado por Fishberg.

c) Rosenstein, citado por Fishberg.

d) Volbard, citado por Fishberg.

d) Volbard, citado por Fisbberg.

e) Traube, citado por Fisbberg.

f) Lichtwitz y Connhein, citado por Fisbberg. 